Research Article

\title{
Compact Stars Admitting Noether Symmetries in Energy-Momentum Squared Gravity
}

\author{
M. Sharif $(\mathbb{D}$ and M. Zeeshan Gul \\ Department of Mathematics, University of the Punjab, Quaid-e-Azam Campus, Lahore 54590, Pakistan \\ Correspondence should be addressed to M. Sharif; msharif.math@pu.edu.pk
}

Received 9 November 2020; Revised 26 November 2020; Accepted 2 December 2020; Published 27 January 2021

Academic Editor: Muhammad Farasat Shamir

Copyright (C) 2021 M. Sharif and M. Zeeshan Gul. This is an open access article distributed under the Creative Commons Attribution License, which permits unrestricted use, distribution, and reproduction in any medium, provided the original work is properly cited.

\begin{abstract}
This paper investigates the geometry of compact stellar objects through the Noether symmetry approach in the energy-momentum squared gravity. This newly developed theory overcomes the problems of big bang singularity and provides the viable cosmological consequences in the early time universe. Moreover, its implications occur in high curvature regime where the deviations of energy-momentum squared gravity from general relativity is confirmed. We consider the minimal coupling model of this modified theory and formulate symmetry generators as well as corresponding conserved quantities. We use conservation relation and apply some suitable initial conditions to evaluate the metric potentials. Finally, we explore some interesting features of the compact objects for appropriate values of the model parameters through numeric analysis. It is found that compact stellar objects in this particular framework depend on the model parameters as well as conserved quantities. We conclude that Noether symmetries generate solutions that are consistent with the astrophysical observational data and hence confirm the viability of this procedure.
\end{abstract}

\section{Introduction}

Noether symmetry approach is recognized as the most efficient method to investigate the analytic solutions that help to find the conserved parameters of the field equations corresponding to symmetry generators. The main motivation comes from various conservation laws (energy, momentum, angular momentum, etc.) which are outcomes of some kind of symmetry being present in a system. The conservation laws are the key factors in the study of various physical processes and familiar Noether theorem, which implies that every differentiable symmetry of the action leads to the law of conservation. This theorem is significant because it provides a correlation between conserved quantities and symmetries of a physical system [1]. A lot of fascinating work has been done in this background [2-5].

Modified gravitational theories are considered as the most favorable and propitious techniques to uncover the cosmic mysteries. Such theories can be formulated by adding the functions of curvature invariants in the geometric part of the Einstein-Hilbert action. The natural modification is obtained by replacing the Ricci scalar $(R)$ with its generic function in the Einstein-Hilbert action so-called $f(R)$ theory of gravity. There has been a crucial literature [6-8] available to understand the viable characteristics of this gravity. This theory has further been generalized by introducing some couplings between curvature invariant and the matter part. These couplings describe different cosmic eras and the rotation curves of galaxies. Such interactions also yield nonconserved stress-energy tensor indicating the existence of an additional force. These coupling models are the key aspects to understand the cosmic accelerated expansion and dark matter/dark energy interactions [9]. The nonminimal coupling between the curvature invariant and matter Lagrangian $\left(L_{m}\right)$ has been established in [10] known as $f\left(R, L_{m}\right)$ gravity. Harko et al. [11] formulated such coupling in $f(R)$ theory referred to as $f(R, T)$ gravity (T represents the trace of stress-energy tensor). A more generic theory in which matter is nonminimally coupled to geometry was proposed in [12], referred to as $f\left(R, T, R_{\mu \nu} T^{\mu \nu}\right)$ 
theory, where $R_{\mu \nu}$ is the Ricci tensor and $T_{\mu \nu}$ demonstrates the stress-energy tensor. One such modifications gave rise to $f\left(R, T^{\phi}\right)$ theory, where $\phi$ defines the scalar field [13].

The presence of singularities can be considered a major problem in general relativity (GR) because of its prediction at high energy level, where GR is no longer valid due to the expected quantum impacts. However, there is no specific formalism for quantum gravity. In this regard, energymomentum squared gravity (EMSG) is considered as the most favorable and prosperous technique which resolves the singularity of the big bang in nonquantum description. This modification of GR is formulated by adding the analytic function $T_{\mu \nu} T^{\mu \nu}$ in the generic action which is also referred as $f\left(R, \mathbf{T}^{2}\right)$ gravity where $T_{\mu \nu} T^{\mu \nu}$ is denoted by $\mathbf{T}^{2}$ [14]. It provides the contribution of squared terms $\left(\rho^{2}, p^{2}\right.$, and $\rho p$ where $\rho$ and $p$ are the matter variables) in the field equations that are used to explore the various fascinating cosmological consequences. This theory has a regular bounce with minimum scale factor $\left(a_{\mathrm{min}}\right)$ and finite maximum energy density $\left(\rho_{\max }\right)$ at early times. Consequently, it can solve the singularity of the big bang with a classical prescription. The cosmological constant does not play a crucial role in the background of the standard cosmological model. The repulsive nature of the cosmological constant supports to resolve singularity only after the matter-dominated era in the EMSG. It is worthwhile to mention here that this theory overcomes the spacetime singularity but does not change the cosmological evolution.

Several researchers have carried out further studies on this theory. Board and Barrow [15] investigated the range of exact solutions for isotropic spacetime, presence of singularities, cosmic accelerated expansion, and evolution with a particular model of this theory. Nari and Roshan [16] studied physical viability and stability of compact stars in this framework. Morares and Sahoo [17] studied nonexotic matter wormholes while Akarsu [18] explored possible constraints from neutron stars in the same frame. Bahamonde et al. [19] investigated the minimal and nonminimal coupling models of EMSG and observed that these models describe the current cosmic accelerated expansion. There has been a recent literature [20-22] that indicates various cosmological applications of this modified theory. It is evident from the above set of literature that EMSG requires more focus, and hence motivations to analyze such theory are very high. There are many open issues that can be studied and this would add as well to improve our current knowledge about different modified theories of gravity.

Noether symmetries have various important applications in modified gravitational theories. Capozziello et al. [23] found static and nonstatic spherical solutions through Noether symmetry technique in $f(R)$ theory. Roshan and Shojai [24] investigated cosmological models of $f(R, G)$ theory ( $G$ is the Gauss-Bonnet invariant) by adopting Noether symmetry technique. Hussain et al. [25] studied the Noether gauge symmetry approach in $f(R)$ theory. Kucukakca [26] analyzed this approach in scalar-tensor teleparallel theory to explore some physically viable cosmological models. Bahamonde [27] investigated the wormhole geometry through the Noether symmetry approach in the same gravity. Sharif and Fatima [28] studied Noether symmetries of FRW spacetime for both dust as well as vacuum cases in $f(G)$ theory. They also formulated exact cosmological models of this gravity and investigated the current cosmic accelerated expansion in terms of scale factor. Shamir and Ahmad [29, 30] used this technique to explore different cosmological models with isotropic and anisotropic matter configurations in the background of $f(G, T)$ theory. Sharif and his collaborators [31-37] analyzed accelerated expansion and evolution of the universe by using this approach. There has been a recent literature [38-42] that indicates various cosmological applications of this approach in various modified theories of gravity.

The attributes and outcomes of self-gravitating objects have great interest for the researchers because of their fascinating features and relativistic geometries in astrophysics as well as cosmology. The final outcome of this phenomenon is the gravitational collapse which is responsible for the formation of new celestial objects named as compact stars. Such compact objects are assumed to be very dense due to extensive masses and short radii. These dense objects may be well described by GR and modified theories [43-45]. Abbas [46] examined the equilibrium state of compact objects and also analyzed their physical attributes in modified Gauss-Bonnet gravity. Zubair and Abbas [47] analyzed the geometry of compact stars with anisotropic matter configuration in $f(R)$ theory. Recently, Shamir and $\mathrm{Naz}$ [39] studied the geometry of compact objects through the Noether symmetry approach in $f(G)$ gravity. The impact of modified theories is well-known to analyze the geometry of compact stars and matter configuration at large densities [48-52].

Since EMSG is established to overcome the singularities, it is significant to analyze the inner region of massive objects where energy factor is quite strong to see the deviations of EMSG from GR. In this paper, we formulate Noether symmetry generators and corresponding conserved quantities for a minimal coupling model of EMSG, i.e., $f\left(R, \mathbf{T}^{2}\right)=\alpha R^{n}+\beta\left(\mathbf{T}^{2}\right)^{m}, n, m \neq 0,1$, and $\alpha, \beta \neq 0$ [19]. We then discuss some salient features of compact objects such as effective matter variables, energy conditions, compactness parameter, gravitational redshift, stability against equilibrium forces, and sound speed for particular values of the model parameters. The paper is organized as follows: in Section 2, we formulate the field equations of static spherical system in the background of EMSG. Section 3 gives a brief description of Noether symmetry technique. In Section 4, we find the expression of metric potentials by using conserved quantities with suitable initial conditions. Section 5 is devoted to analyze some physical characteristics of compact star to explore the viability of the model through graphs. We summarize and discuss the results in the last section.

\section{Basic Formalism of Energy-Momentum Squared Gravity}

In this section, we formulate the field equations for EMSG in the presence of perfect fluid. The action for this theory is determined as follows [14]: 


$$
S=\frac{1}{2 \kappa^{2}} \int f\left(R, \mathbf{T}^{2}\right) \sqrt{-g} d^{4} x+\int L_{m} \sqrt{-g} d^{4} x,
$$

where $\kappa^{2}$ and $g$ represent the coupling constant and determinant of the line element, respectively. We consider coupling constant as a unity for the sake of simplicity. The action indicates that this theory has extra degrees of freedom. Therefore, due to additional force and matter-dominated era, it is expected that some useful consequences would be obtained to study the current cosmic issues in this gravity. The variation of the action corresponding to the metric tensor leads to the following field equations:

$$
R_{\mu \nu} f_{R}+g_{\mu \nu} \square f_{R}-\nabla_{\mu} \nabla_{\nu} f_{R}-\frac{1}{2} g_{\mu \nu} f=T_{\mu \nu}-\Theta_{\mu \nu} f_{\mathrm{T}^{2}},
$$

where $\quad \square=\nabla_{\mu} \nabla^{\mu}, \quad f \equiv f\left(R, \mathbf{T}^{2}\right), \quad f_{\mathbf{T}^{2}}=\left(\partial f / \partial \mathbf{T}^{2}\right)$, $f_{R}=(\partial f / \partial R)$, and

$$
\Theta_{\mu \nu}=-2 L_{m}\left(T_{\mu \nu}-\frac{1}{2} g_{\mu \nu} T\right)-4 \frac{\partial^{2} L_{m}}{\partial g^{\mu \nu} \partial g^{\xi \eta}} T^{\xi \eta}-T T_{\mu \nu}+2 T_{\xi}^{\mu} T_{\nu \xi} .
$$

It is noted that for $f\left(R, \mathbf{T}^{2}\right)=f(R)$, the field equations of this gravity reduce to $f(R)$ theory while GR is recovered when $f\left(R, \mathbf{T}^{2}\right)=R$.

We assume matter distribution as a perfect fluid:

$$
T_{\mu \nu}^{m}=\left(\rho_{m}+p_{m}\right) U_{\mu} U_{\nu}+g_{\mu \nu} p_{m}
$$

where $\rho_{m}, p_{m}$, and $U_{\mu}$ demonstrate the energy density, pressure, and four-velocity, respectively. The Lagrangian corresponding to matter distribution (4) is defined as $L_{m}=\mathrm{p}_{m}$, and manipulating equation (3), we obtain

$$
\Theta_{\mu \nu}=-\left(3 p_{m}^{2}+\rho_{m}^{2}+4 p_{m} \rho_{m}\right) U_{\mu} U_{v} .
$$

Rearranging equation (2), we have

$$
\mathscr{G}_{\mu \nu}=\frac{1}{f_{R}}\left(T_{\mu \nu}^{c}+T_{\mu \nu}^{m}\right)=T_{\mu \nu}^{\mathrm{eff}},
$$

where $\mathscr{G}_{\mu \nu}=R_{\mu \nu}-(1 / 2) R g_{\mu \nu}$ is the Einstein tensor, $T_{\mu \nu}^{c}$ is the additional effects of EMSG named as correction terms, and $T_{\mu \nu}^{\text {eff }}$ determines the effective stress-energy tensor expressed as

$$
T_{\mu \nu}^{\mathrm{eff}}=\frac{1}{f_{R}}\left\{T_{\mu \nu}^{m}-g_{\mu \nu} \square f_{R}+\nabla_{\mu} \nabla_{\nu} f_{R}-\Theta_{\mu \nu} f_{\mathrm{T}^{2}}+\frac{1}{2} g_{\mu \nu}\left(f-R f_{R}\right)\right\} .
$$

In order to study the characteristics of compact stars, we consider static spherical spacetime as follows [53]:

$$
\mathrm{d} s^{2}=-e^{\lambda(r)} \mathrm{d} t^{2}+e^{9(r)} \mathrm{d} r^{2}+r^{2} d \theta^{2}+r^{2} \sin ^{2} \theta d \phi^{2} .
$$

The respective field equations turn out to be

$$
\begin{aligned}
& \rho^{\text {eff }}=\frac{1}{f_{R}}\left\{\rho_{m}-\frac{1}{2}\left(f-R f_{R}\right)+\left(3 p_{m}^{2}+\rho_{m}^{2}+4 p_{m} \rho_{m}\right) f_{\mathrm{T}^{2}}+e^{-\vartheta}\left\{f_{R}^{\prime \prime}-\left(\frac{\lambda^{\prime}}{2}-\frac{2}{r}\right) f_{R}^{\prime}\right\}\right\}, \\
& p^{\text {eff }}=\frac{1}{f_{R}}\left\{p_{m}+\frac{1}{2}\left(f-R f_{R}\right)-e^{-\vartheta}\left(\frac{\lambda^{\prime}}{2}+\frac{2}{r}\right) f_{R}^{\prime}\right\} .
\end{aligned}
$$

These equations are highly nonlinear as well as complicated due to the presence of multivariate function and its derivatives. We consider the Noether symmetry approach to obtain the analytic solutions of $f\left(R, \mathbf{T}^{2}\right)$ field equations. The conservation law does not hold in this theory, but we obtain conserved quantities in the background of the Noether symmetry approach. These are helpful to obtain physically viable solutions and hence analyze the geometry of compact objects.

\section{Pointlike Lagrangian and Noether Symmetry}

Noether symmetry provides a fascinating procedure to develop new cosmological models and related structures in modified gravitational theories. Here, we formulate the pointlike Lagrangian for static spherical spacetime in the background of EMSG. We determine the corresponding equations by using Noether symmetry technique. This method provides a unique nature of the vector field within the tangent space associated with it. Hence, the vector field behaves as a symmetry generator and gives conserved quantities which are then useful to examine exact solutions of the modified field equations.
The canonical form of action (1) gives

$$
S=\int \mathscr{L}\left(\lambda, \vartheta, R, \mathbf{T}^{2}, \lambda^{\prime}, \vartheta^{\prime}, R^{\prime},\left(\mathbf{T}^{2}\right)^{\prime}\right) \mathrm{d} r .
$$

Using Lagrange multiplier approach, we have

$$
S=\int \sqrt{-g}\left\{f-(R-\bar{R}) \mathscr{V}_{1}-\left(\mathbf{T}^{2}-\overline{\mathbf{T}}^{2}\right) \mathscr{V}_{2}+p_{m}(\lambda, \vartheta)\right\} \mathrm{d} r,
$$

where

$$
\begin{aligned}
\sqrt{-g} & =e^{(\lambda+9 / 2)} r^{2}, \\
\overline{\mathbf{T}}^{2} & =3 p_{m}^{2}+\rho_{m}^{2}, \\
\mathscr{V}_{1} & =f_{R}, \\
\mathscr{V}_{2} & =f_{\mathrm{T}^{2},} \\
\bar{R} & =-\frac{1}{e^{9}}\left(\lambda^{\prime \prime}+\frac{\lambda^{\prime 2}}{2}+\frac{2 \lambda^{\prime}}{r}-\frac{29^{\prime}}{r}-\frac{\lambda^{\prime} \vartheta^{\prime}}{2}-\frac{2 e^{9}}{r^{2}}+\frac{2}{r^{2}}\right) .
\end{aligned}
$$


We see that if $R-\bar{R}=0$ and $\mathbf{T}^{2}-\overline{\mathbf{T}}^{2}=0$, then the above action reduces to action (1). Substituting the values from equation (12) in (11) and eliminating the boundary terms with the help of integration by parts, we have

$$
\begin{aligned}
\mathscr{L}\left(\lambda, \vartheta, R, \mathbf{T}^{2}, \lambda^{\prime}, \vartheta^{\prime}, R^{\prime},\left(\mathbf{T}^{2}\right)^{\prime}\right)= & r^{2} e^{(\lambda+9 / 2)}\left(f+p_{m}-R f_{R}+\frac{2 f_{R}^{2}}{r}+f_{T^{2}}\left(3 p_{m}^{2}+\rho_{m}^{2}-\mathbf{T}^{2}\right)\right) \\
& +r^{2} e^{(\lambda-9 / 2)}\left\{\left(\frac{2 \vartheta^{\prime}}{r}-\frac{2}{r^{2}}\right) f_{R}+\lambda^{\prime} R^{\prime} f_{R R}+\lambda^{\prime}\left(\mathbf{T}^{2}\right)^{\prime} f_{R \mathbf{T}^{2}}\right\} .
\end{aligned}
$$

The Euler-Lagrange equations are given as follows:

$$
\frac{\partial \mathscr{L}}{\partial q^{i}}-\frac{d}{\mathrm{~d} r}\left(\frac{\partial \mathscr{L}}{\partial q^{i^{\prime}}}\right)=0,
$$

where $q^{i}$ represents the generalized coordinates of $n$-dimensional space. By using Lagrangian (13), equation (14) turns out to be

$$
\begin{aligned}
& f-R f_{R}+p_{m}+f_{\mathrm{T}^{2}}\left(3 p_{m}^{2}+\rho_{m}^{2}+12 p_{m} p_{m_{, \lambda}}+4 \rho \rho_{m_{, \lambda}}-\mathbf{T}^{2}\right)+2 p_{m_{,},} \\
& +\frac{1}{e^{\vartheta}}\left\{\left(\frac{2 \vartheta^{\prime}}{r}+\frac{2 e^{\vartheta}}{r^{2}}-\frac{2}{r^{2}}\right) f_{R}+\left(\vartheta^{\prime} R^{\prime}-2 R^{\prime \prime}-\frac{4 R^{\prime}}{r}\right) f_{R R}\right. \\
& +\left(\vartheta^{\prime}\left(\mathbf{T}^{2}\right)^{\prime}-2\left(\mathbf{T}^{2}\right)^{\prime \prime}-\frac{4\left(\mathbf{T}^{2}\right)^{\prime}}{r}\right) f_{R \mathbf{T}^{2}}-2{R^{\prime}}^{2} f_{R R R} \\
& \left.-4 R^{\prime}\left(\mathbf{T}^{2}\right)^{\prime} f_{R R \mathbf{T}^{2}}-2\left(\mathbf{T}^{2}\right)^{\prime} f_{R \mathbf{T}^{2} \mathbf{T}^{2}}\right\}=0, \\
& f-R f_{R}+p_{m}+f_{\mathbf{T}^{2}}\left(3 p_{m}^{2}+\rho_{m}^{2}+12 p_{m} p_{m, 9}+4 \rho \rho_{m_{, 9}}-\mathbf{T}^{2}\right) \\
& +2 p_{m_{, 9}}+\frac{1}{e^{\vartheta}}\left\{\left(\frac{2 e^{\vartheta}}{r^{2}}-\frac{2 \lambda^{\prime}}{r}-\frac{2}{r^{2}}\right) f_{R}-\left(\lambda^{\prime} R^{\prime}+\frac{4 R^{\prime}}{r}\right) f_{R R}\right. \\
& \left.-\left(\lambda^{\prime}\left(\mathbf{T}^{2}\right)^{\prime}+\frac{4\left(\mathbf{T}^{2}\right)^{\prime}}{r}\right) f_{R \mathbf{T}^{2}}\right\}=0 \\
& e^{\vartheta}\left\{\left(R-\frac{2}{r^{2}}\right) f_{R R}-\left(3 p_{m}^{2}+\rho_{m}^{2}-\mathbf{T}^{2}\right) f_{R \mathbf{T}^{2}}\right\}+\left(\lambda^{\prime \prime}+\frac{\lambda^{\prime 2}}{2}+\frac{2 \lambda^{\prime}}{r}-\frac{2 \vartheta^{\prime}}{r}-\frac{\lambda^{\prime} \vartheta^{\prime}}{2}+\frac{2}{r^{2}}\right) f_{R R}=0, \\
& e^{\vartheta}\left\{\left(R-\frac{2}{r^{2}}\right) f_{R \mathbf{T}^{2}}-\left(3 p_{m}^{2}+\rho_{m}^{2}-\mathbf{T}^{2}\right) f_{\mathbf{T}^{2} \mathbf{T}^{2}}\right\}+\left(\lambda^{\prime \prime}+\frac{\lambda^{\prime 2}}{2}+\frac{2 \lambda^{\prime}}{r}-\frac{2 \vartheta^{\prime}}{r}-\frac{\lambda^{\prime} \vartheta^{\prime}}{2}+\frac{2}{r^{2}}\right) f_{R \mathbf{T}^{2}}=0 .
\end{aligned}
$$

The corresponding Hamiltonian, $H=q^{i^{\prime}}\left(\partial \mathscr{L} / \partial q^{i^{\prime}}\right)-\mathscr{L}$, turns out to be

$$
\begin{aligned}
H= & -e^{(\lambda-\vartheta / 2)} r^{2}\left\{e^{\vartheta}\left(f-R f_{R}+\left(3 p_{m}^{2}+\rho_{m}^{2}-\mathbf{T}^{2}\right) f_{\mathbf{T}^{2}}+p_{m}+\frac{2 f_{R}}{r^{2}}\right)\right. \\
& \left.-\frac{2 f_{R}}{r^{2}}-\lambda^{\prime} R^{\prime} f_{R R}-\lambda^{\prime}\left(\mathbf{T}^{2}\right)^{\prime} f_{R \mathbf{T}^{2}}\right\} .
\end{aligned}
$$

The generators of Lagrangian (13) are considered as follows:

$$
Y=\varrho \frac{\partial}{\partial r}+\zeta^{i} \frac{\partial}{\partial q^{i}},
$$

where $\varrho \equiv \varrho\left(\lambda, \vartheta, R, \mathbf{T}^{2}\right)$ and $\zeta^{i} \equiv \zeta^{i}\left(\lambda, \vartheta, R, \mathbf{T}^{2}\right)(i=1,2,3,4)$ are unknown coefficients of the vector field $Y$. The Lagrangian must fulfill the condition of invariance for the vector field over the tangent space to assure the existence of 
Noether symmetries. In this regard, $Y$ acts as a symmetry generator that constructs the conserved quantities. The condition of invariance can be expressed as follows:

$$
Y^{[1]} \mathscr{L}+(D \varrho) \mathrm{L}=D \psi,
$$

where $\psi$ represents the boundary term, $Y^{[1]}$ is the first order prolongation, and $D$ demonstrates the total rate of change. This can also be expressed as follows:

$$
\begin{gathered}
Y^{[1]}=Y+\zeta^{i^{i}} \frac{\partial}{\partial q^{i^{i}}}, \\
D=\frac{\partial}{\partial r}+q^{i^{i}} \frac{\partial}{\partial q^{i^{i}}},
\end{gathered}
$$

where $\zeta^{i^{\prime}}=D \zeta^{i^{\prime}}-q^{i^{\prime}} D \psi$. The first integral of motion corresponds to Noether symmetry generator $Y$ which is determined as follows:

$$
I=-\varrho H+\zeta \frac{\partial \mathscr{L}}{\partial q^{i}}-\psi
$$

This is the most significant part of Noether symmetries which is also known as a conserved quantity. It is interesting to mention here that the first integral plays a remarkable role to obtain physically viable solutions.

The first integrals of motion are the main factors to determine the characteristics of massive objects in modified gravitational theories. By considering equation (18) and comparing the coefficients, we have a set of partial differential equations named as determining equations. In this case, we obtain the following system of equations:

$$
\begin{aligned}
& \varrho_{, \lambda}=0, \varrho_{, 9}=0, \varrho_{, R}=0, \varrho_{, \mathrm{T}^{2}}=0, \\
& \lambda_{, 9}=0, \lambda_{, R}=0, \lambda_{, \mathrm{T}^{2}}=0,
\end{aligned}
$$

$\zeta_{, 9}^{3} f_{R R}+\zeta_{, 9}^{4} f_{R \mathrm{~T}^{2}}=0, \zeta_{, \lambda}^{3} f_{R R}+\zeta_{, \lambda}^{4} f_{R \mathrm{~T}^{2}}=0$,

$$
\begin{aligned}
& e^{\lambda+9 / 2} r^{2}\left\{f-R f_{R}+p_{m}+f_{\mathrm{T}^{2}} 3 p_{m}^{2}+\rho_{m}^{2}-\mathrm{T}^{2}+\frac{2 f_{R}}{r^{2}}\right. \\
& \times \frac{\zeta^{1}+\zeta^{2}}{2}+\varrho_{, r}+\zeta^{1} f_{\mathrm{T}^{2}} 6 \mathrm{p}_{m} \mathrm{p}_{m_{,}}+2 \rho \rho_{m_{\lambda}}+\mathrm{p}_{m_{, \lambda}} \\
& +\zeta^{2} f_{\mathrm{T}^{2}} 6 p_{m} p_{m_{,}}+2 \rho \rho_{m, 9}+p_{m, 9}-\zeta^{4} f_{R R} R-2 r^{-2} \\
& +f_{R \mathrm{~T}^{2}} 3 p_{m}^{2}+\rho_{m}^{2}-\mathrm{T}^{2}-\zeta^{5} f_{\mathrm{T}^{2} \mathrm{~T}^{2}} 3 p_{m}^{2}+\rho_{m}^{2}-\mathrm{T}^{2} \\
& \left.+f_{R \mathrm{~T}^{2}} R-2 r^{-2}+\frac{2 f_{R}}{\mathrm{re}^{9}} \psi_{, r}\right\}-\psi_{, r}=0 .
\end{aligned}
$$


Noether symmetry method minimizes the complexity of the system and helps to determine the exact solutions. However, it is complicated to derive a nontrivial solution without taking any specific EMSG model. The analysis of compact stars through Noether symmetry technique in the curvature-matter coupling model would provide interesting consequences. We investigate the presence of symmetry generators with corresponding conserved quantities and investigate structure of compact objects for $f\left(R, \mathbf{T}^{2}\right)$ gravity model. The minimal model is defined as follows:

$$
f\left(R, \mathbf{T}^{2}\right)=\alpha R^{n}+\beta\left(\mathbf{T}^{2}\right)^{m} \text {. }
$$

This model determines a higher complexity of the phase space characteristics with three main eras of the universe (radiation-dominated, matter-dominated, and de Sitter) and solutions exhibit accelerated expansions. We consider $m=2=n$ for the sake of convenience.

It is worthwhile to explore perfect fluid as it explains exact matter of various celestial objects like stars, galaxies, etc. The cosmic matter configuration can also be examined by dust fluid only when negligible amount of radiations is present. The interaction of radiations with dust particles supports to develop compact objects. In the following equation, we examine features of compact stars and derive exact solutions of the EMSG model for dust matter distribution, i.e., $T_{\mu \nu}^{m}=\rho_{m} U_{\mu} U_{v}$. The simultaneous solutions of equations (21)-(30) yield

$$
\begin{aligned}
\zeta^{1}= & c 1, \\
\zeta^{2}= & c 1+2 c 2, \\
\zeta^{3}= & R c 2, \\
\varrho & =c 3 r, \\
\zeta^{4}= & 0=\psi, \\
\rho_{m}= & \left\{\left(R \alpha \beta\left(R c 1 r^{2}+3 R c 2 r^{2}+R c 3 r^{2}-4 c 1-8 c 2-4 c 3\right)\right.\right. \\
& \left.\times(c 1+c 2+c 3))^{(1 / 2)} \beta r(c 1+c 2+c 3)\right\}^{(1 / 2)}\{\beta r(c 1+c 2+c 3)\}^{-1},
\end{aligned}
$$

where $c i$ represents the arbitrary constants. The generators of the Noether symmetry and corresponding conserved quantities become

$$
\begin{aligned}
Y_{1}= & \frac{\partial}{\partial \lambda}+\frac{\partial}{\partial \vartheta} \\
Y_{2}= & \frac{\partial}{\partial \vartheta}+R \frac{\partial}{\partial R} \\
Y_{3}= & r \frac{\partial}{\partial r} \\
I_{1}= & 2 R \alpha e^{(\lambda-9 / 2)}\left(4 r+a^{\prime} r^{2}\right), \\
I_{2}= & 2 \alpha e^{(\lambda-9 / 2)}\left(r^{2} R^{\prime}+2 r R\right), \\
I_{3}= & -r^{3} \alpha e^{(\lambda-9 / 2)}\left(2 a^{\prime} R^{\prime}-R^{2}+4 R r^{-2}\right. \\
& \left.+e^{9} r^{2}-4 e^{9} R r^{-2}\right) .
\end{aligned}
$$

\section{Metric Potentials and Boundary Conditions}

The conserved quantities obtained through the Noether symmetry approach play a significant role to investigate various physical characteristics of compact objects. This approach has successfully been executed in axial and spherically symmetric spacetimes $[54,55]$. The solutions at the boundary of compact objects are determined by smooth matching of internal and external geometries. The metric potentials of both interior and exterior spacetimes are joined at the surface boundary through the relationship

$$
e^{\lambda(r)}=e^{-\vartheta(r)} \text {. }
$$

We use the above mentioned conserved quantities to obtain the metric potentials that are helpful to analyze the realistic features of compact objects. Using equation (35) in (33) and (34), it follows

$$
\begin{aligned}
& I_{1}=2 R \alpha e^{\lambda}\left(4 r+a^{\prime} r^{2}\right), \\
& I_{2}=2 \alpha e^{\lambda}\left(r^{2} R^{\prime}+2 r R\right), \\
& I_{3}=-r^{3} \alpha e^{\lambda}\left(2 a^{\prime} R^{\prime}-R^{2}+4 R r^{-2}+e^{-\lambda} r^{2}-4 e^{-\lambda} R r^{-2}\right) .
\end{aligned}
$$

These equations cannot be solved analytically due to their complicated and highly nonlinear nature. Hence, we adopt a numerical method with suitable initial conditions to examine the viable behavior of the metric elements.

In order to check the existence of singularities, we examine the viable behavior of line elements. For a physically realistic and stable cosmological model, the metric potentials must be nonsingular, positive as well as regular inside the geometry of compact objects. The graphical behavior of the metric elements obtained by first, second, and third conserved quantities $\left(I_{1}, I_{2}, I_{3}\right)$ are presented in Figures 1-3, respectively, which shows that the metric elements fulfill all the required conditions. We would like to mention here that for physically viable compact stars, the following conditions must be satisfied:

(i) The effective energy density should be positive inside the stellar object as well as at the surface boundary $\left(\rho^{\text {eff }}>0,0 \leq r \leq \mathscr{R}\right)$.

(ii) The effective pressure should be positive inside the stellar object $\left(p^{\text {eff }}>0\right)$ and should be zero $\left(p^{\text {eff }}=0\right)$ at the surface boundary $(r=\mathscr{R})$.

(iii) The gradient of effective matter variables should be negative for $0 \leq r \leq \mathscr{R}$, i.e., $\left\{\left(d \rho^{\mathrm{eff}} / \mathrm{d} r\right)_{r=0}=0\right.$, $\left(d^{2} \rho^{\text {eff }} / \mathrm{d} r^{2}\right)_{r=0}<0, \quad\left(d p^{\text {eff }} / \mathrm{d} r\right)_{r=0}=0, \quad$ and $\left.\left(d^{2} p^{\text {eff }} / \mathrm{d} r^{2}\right)_{r=0}<0\right\}$. This condition shows that the effective matter variables must be decreasing at the boundary of the surface.

(iv) The sound speed must be less than the speed of the light.

These physical characteristics are important to determine the geometry of compact objects. 


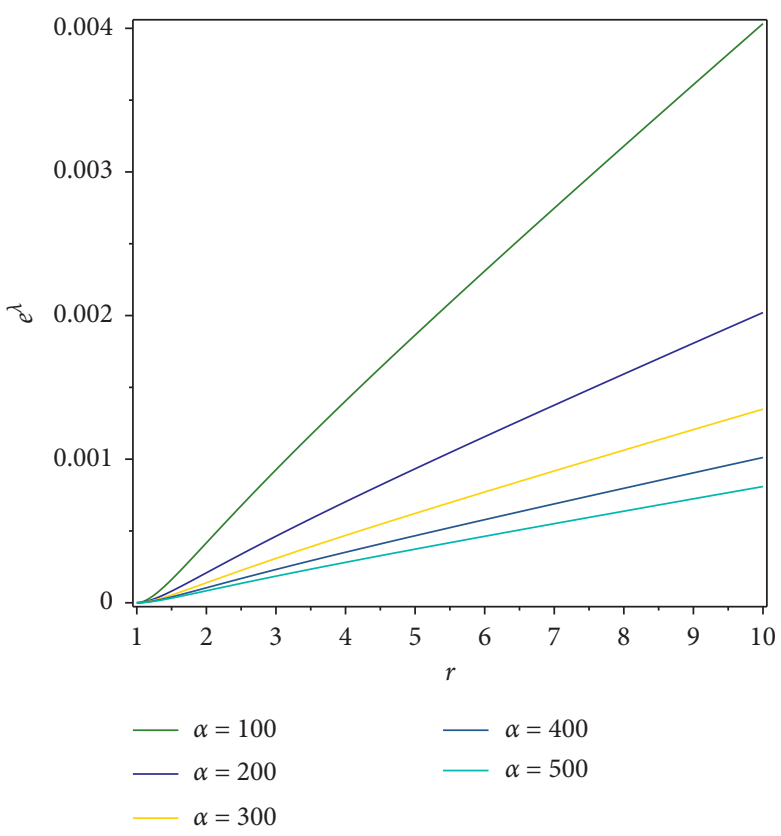

(a)

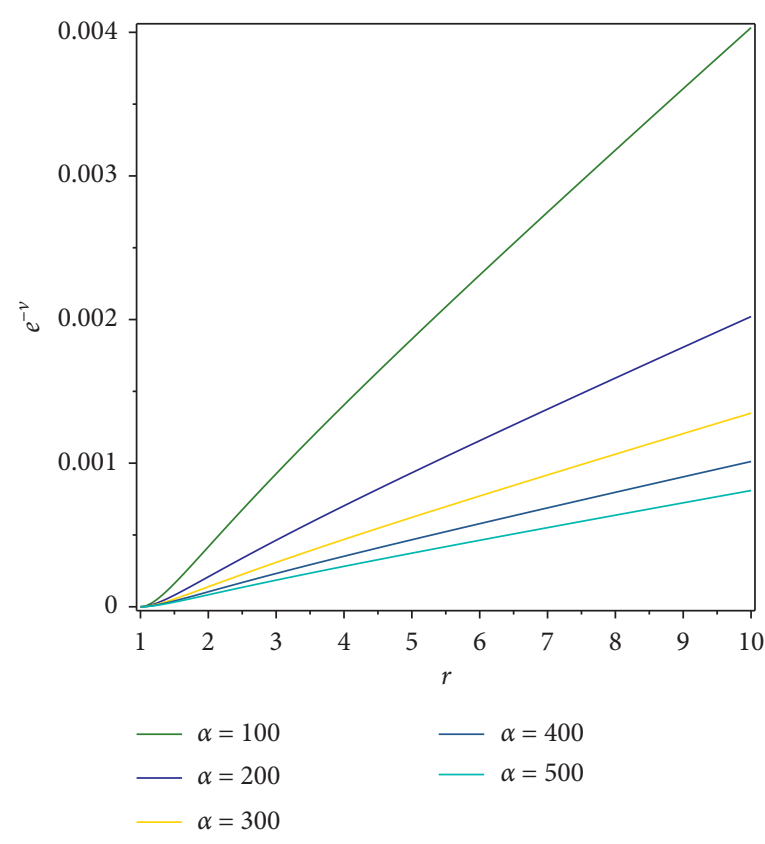

(b)

FIgURE 1: Plots of metric potentials corresponding to $r$.

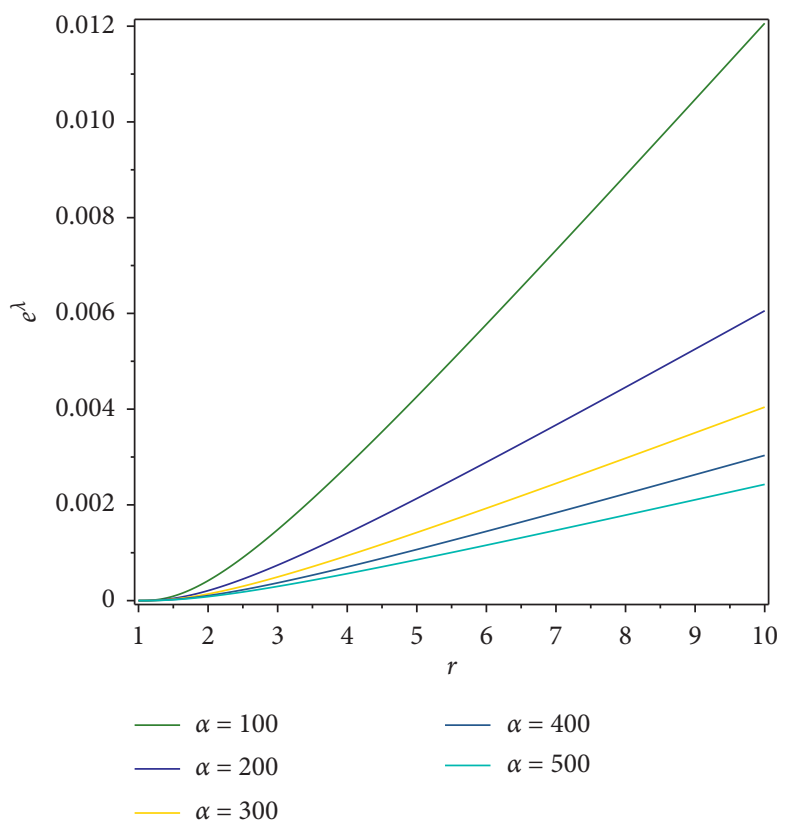

(a)

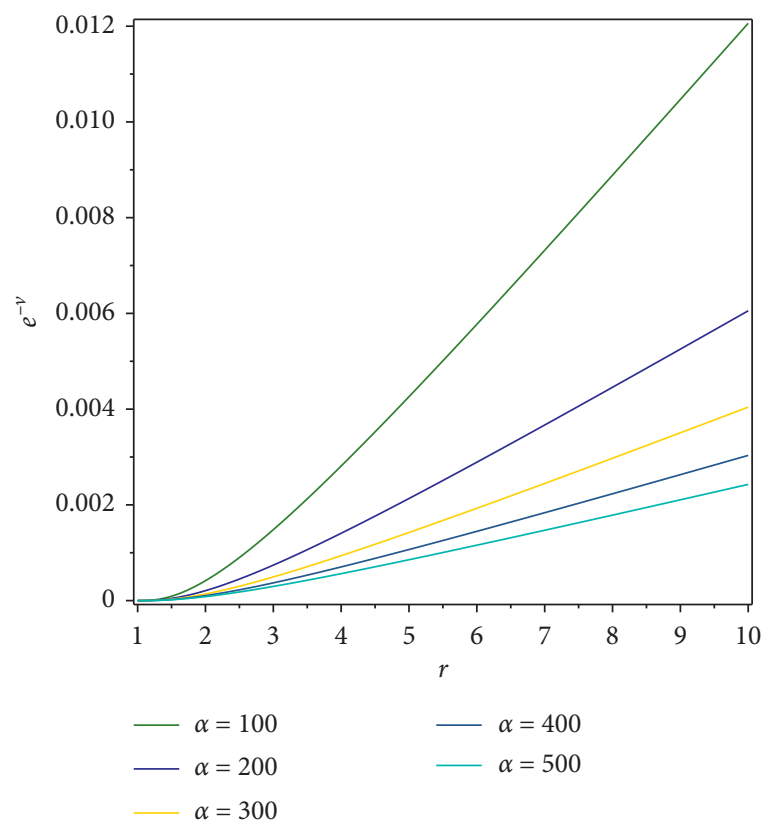

(b)

FIgURe 2: Plots of metric potentials corresponding to $r$. 

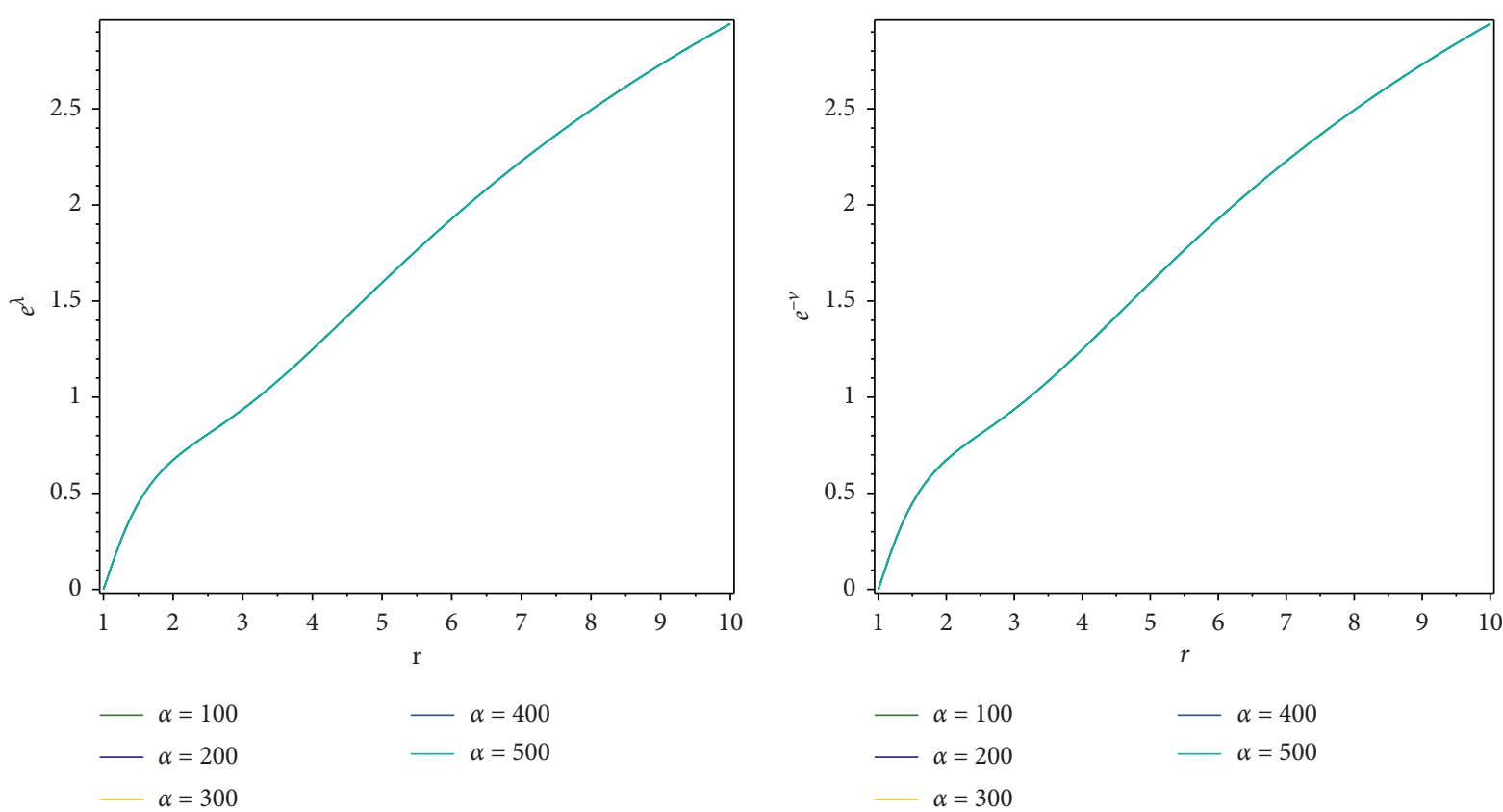

(a)

(b)

Figure 3: Plots of metric potentials corresponding to $r$.

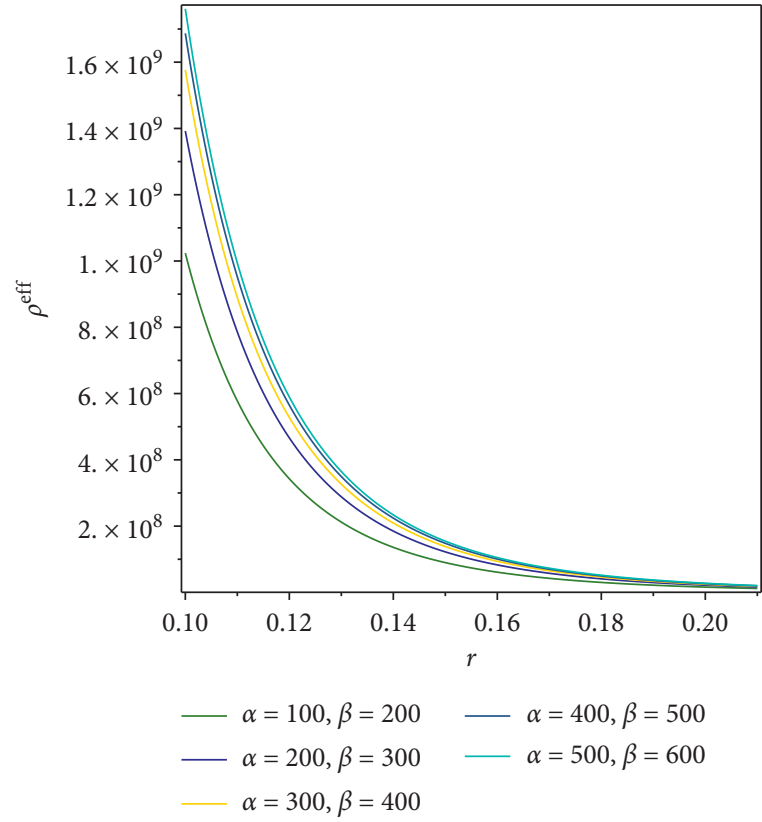

(a)

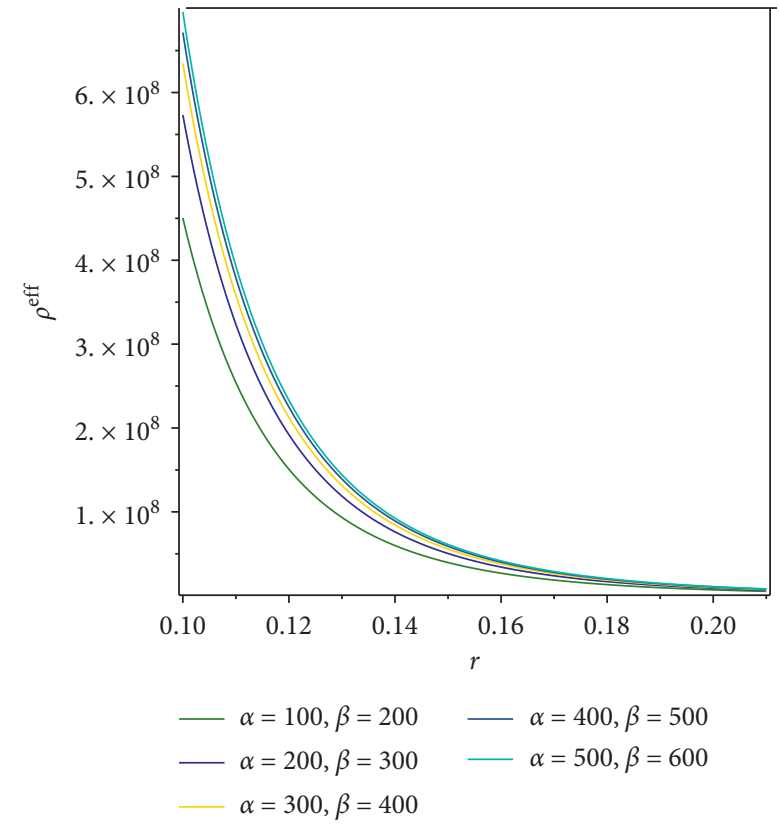

(b)

FIGURE 4: Plots of effective matter variables corresponding to $r$. 


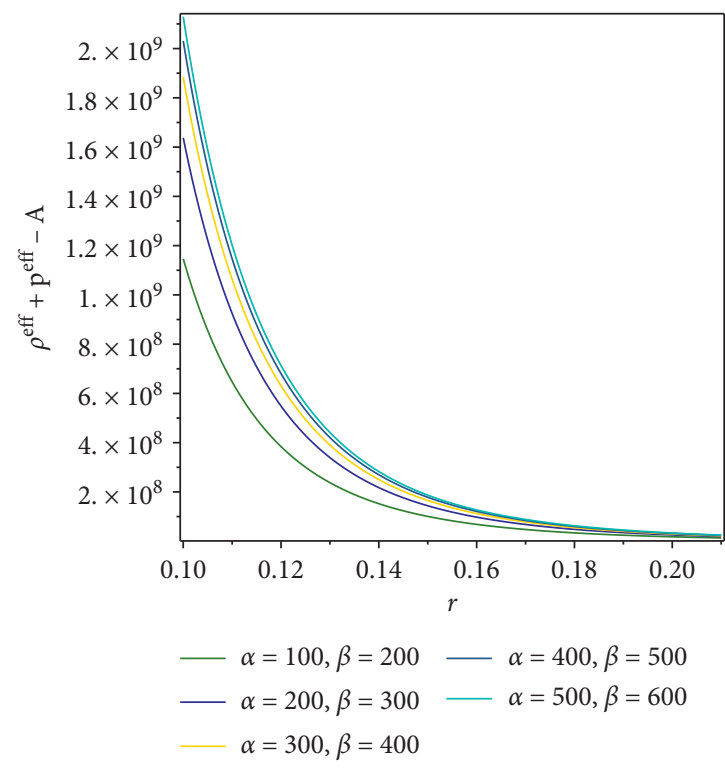

(a)

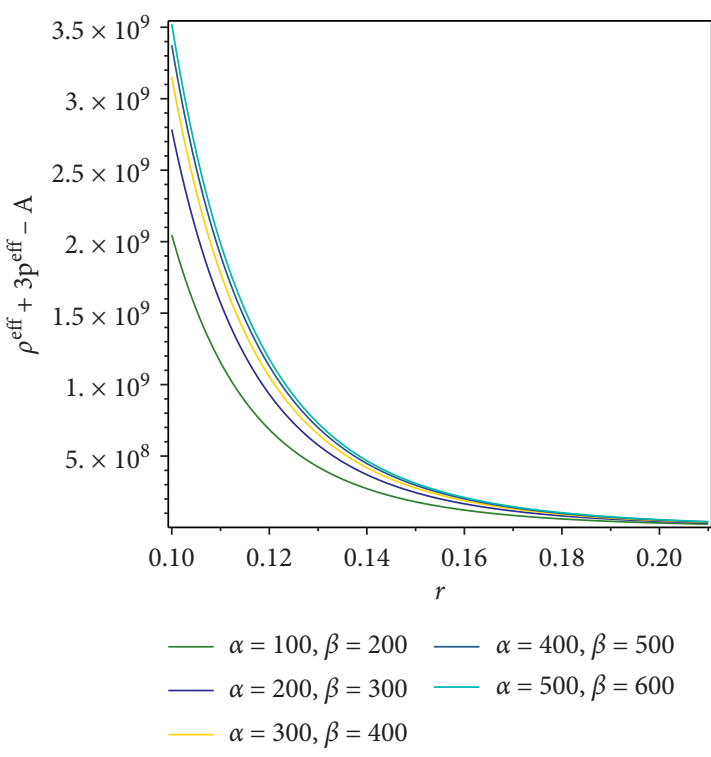

(c)

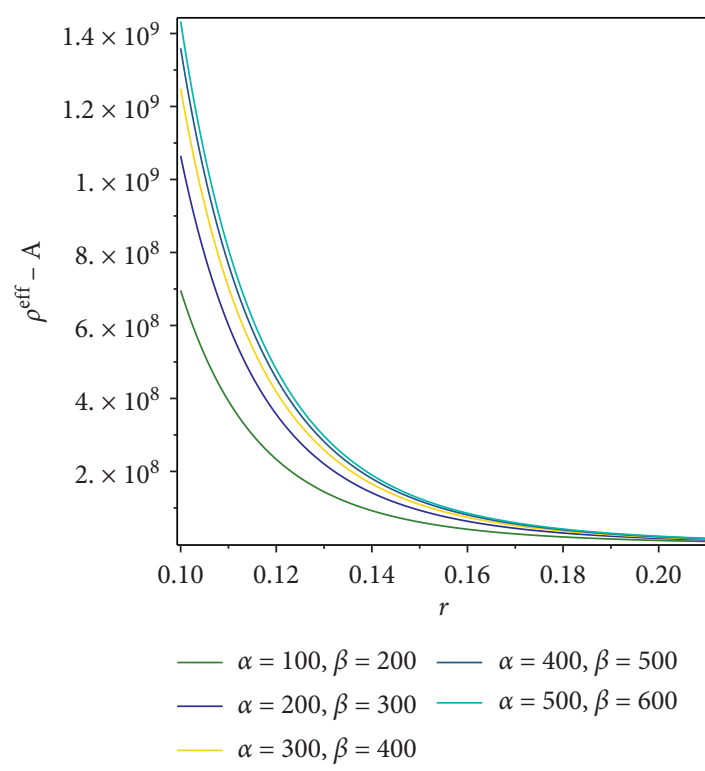

(b)

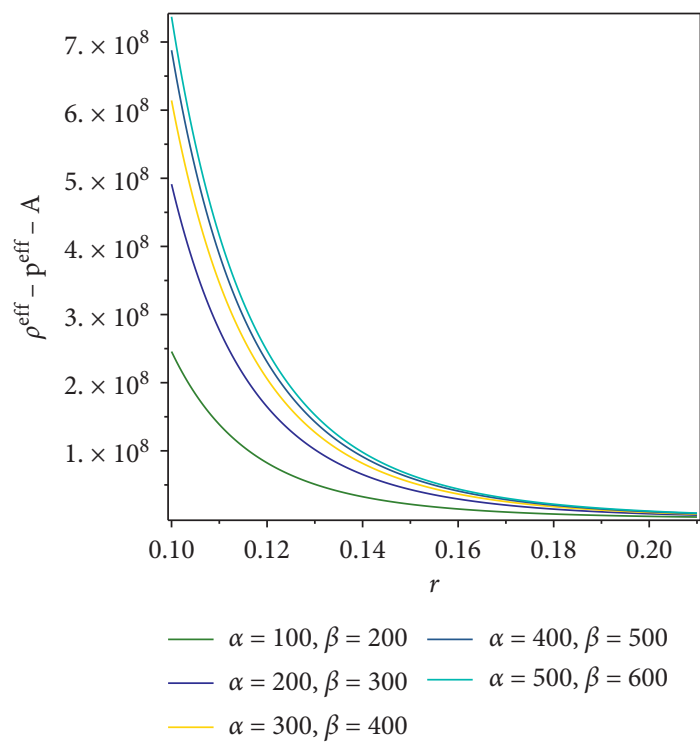

(d)

Figure 5: Plots of energy bounds.

In the following, we discuss physical attributes of compact objects for the metric potentials obtained only by the first conserved quantity $\left(I_{1}\right)$ because the effective matter variables become undefined for the metric potentials obtained by the second conserved quantity $\left(I_{2}\right)$ while the third conserved quantity $\left(I_{3}\right)$ is quite complicated as we could not find the appropriate value of the metric elements.

\section{Physical Characteristics of Compact Objects}

Here, we study physical features of the compact stars through graphical analysis of the effective matter variables, energy bounds, compactness parameters, gravitational redshifts, and stability analysis against equilibrium forces and speed of sound.
5.1. Evolution of the Effective Matter Variables. The effective matter variables inside the compact object should be maximum at the center. For this purpose, we analyze the behavior of compact objects for the range $0 \leq r \leq 10$. We plot the graphs for small radii to have the smooth nature of compact objects. Figure 4 shows that the behavior of effective matter variables is positive and represents the decreasing nature at the boundary of the stellar structure. This assures high compactness of the compact star at the center. In fact, we observe that $\left(d \rho^{\text {eff }} / \mathrm{d} r\right)=0$, $\left(d^{2} \rho^{\text {eff }} / \mathrm{d} r^{2}\right)<0$, and $\left(d p^{\text {eff }} / \mathrm{d} r\right)=0,\left(d^{2} p^{\text {eff }} / \mathrm{d} r^{2}\right)<0$ at $r=$ 0 which determines the compact behavior of the star. Here, we note that stellar geometries depend upon the first integrals of motion. 


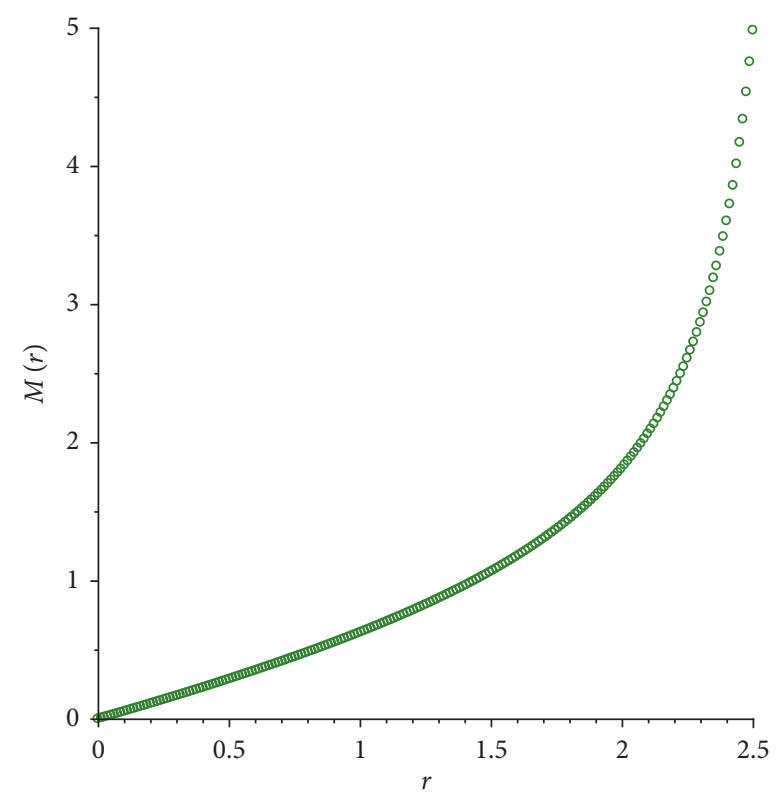

FIGURE 6: Evolution of the mass function with respect to the radial coordinate.

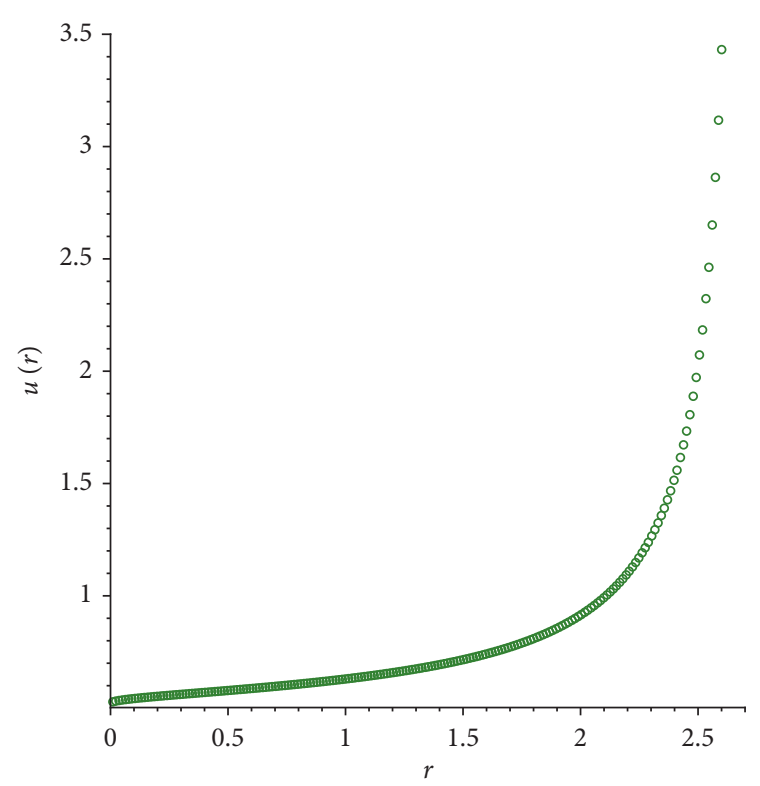

(a)

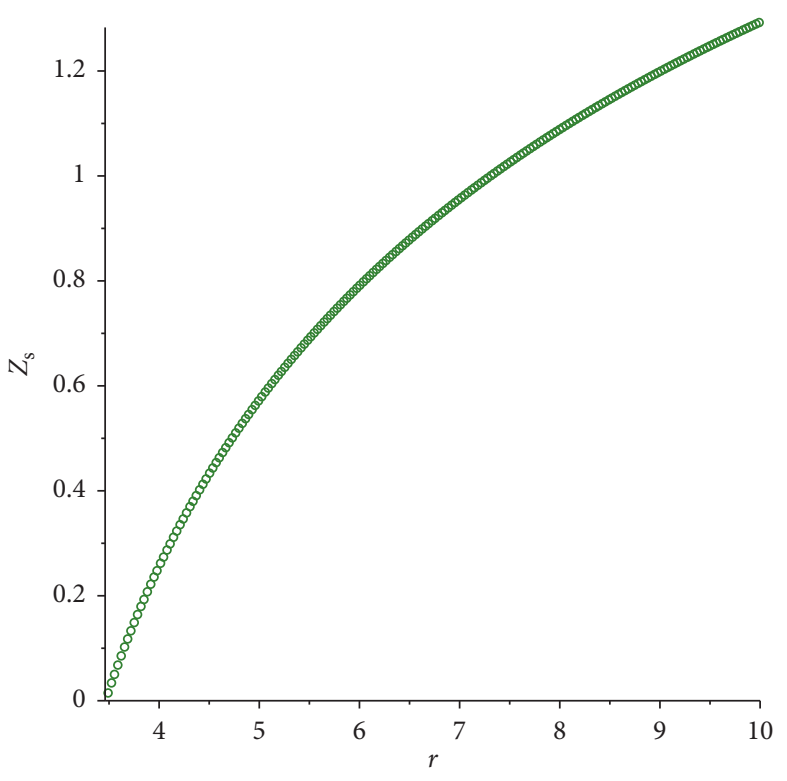

(b)

Figure 7: Plots of the compactness parameter and surface redshift with respect to the radial coordinate.

5.2. Energy Conditions. The energy conditions play a crucial role to investigate the physical existence of cosmological geometries as well as viable matter distribution. For a physically realistic geometry of compact objects, these conditions must be satisfied. These conditions are considered as quite helpful to examine the nature of matter (normal/exotic) inside the geometry of compact stars. These bounds can be categorized into null $(\mathbb{N E} \mathbb{C})$, weak ( $\mathbb{W} \mathbb{E} C$ ), strong $(\mathbb{S E C})$, and dominant $(\mathbb{D E} \mathbb{C})$ energy conditions. In curvature-matter coupled gravity, these bounds are expressed as follows [56]:

$$
\begin{array}{r}
\mathbb{N} \mathbb{E} C: \rho^{\text {eff }}+p^{\text {eff }}-A \geq 0, \\
\mathbb{V} \mathbb{E} \mathbb{C}: \rho^{\text {eff }}-A \geq 0, \rho^{\text {eff }}+p^{\text {eff }}-A \geq 0, \\
\mathbb{S E C}: \rho^{\text {eff }}+p^{\text {eff }}-A \geq 0, \rho^{\text {eff }}+3 p^{\text {eff }}-A \geq 0, \\
\mathbb{D} \mathbb{E} \mathbb{C}: \rho^{\text {eff }}-A \geq 0, \rho^{\text {eff }} \pm p^{\text {eff }}-A \geq 0,
\end{array}
$$

where $A=\left(1 / 4 e^{9}\right)\left(\lambda^{\prime 2}+2 \lambda^{\prime \prime}+4 \lambda^{\prime} r^{-1}-\lambda^{\prime} 9^{\prime}\right)$ determines an acceleration term that exists due to the additional impacts of curvature-matter coupled gravity. For the physically viable geometry, the energy density must be finite as well as positive 


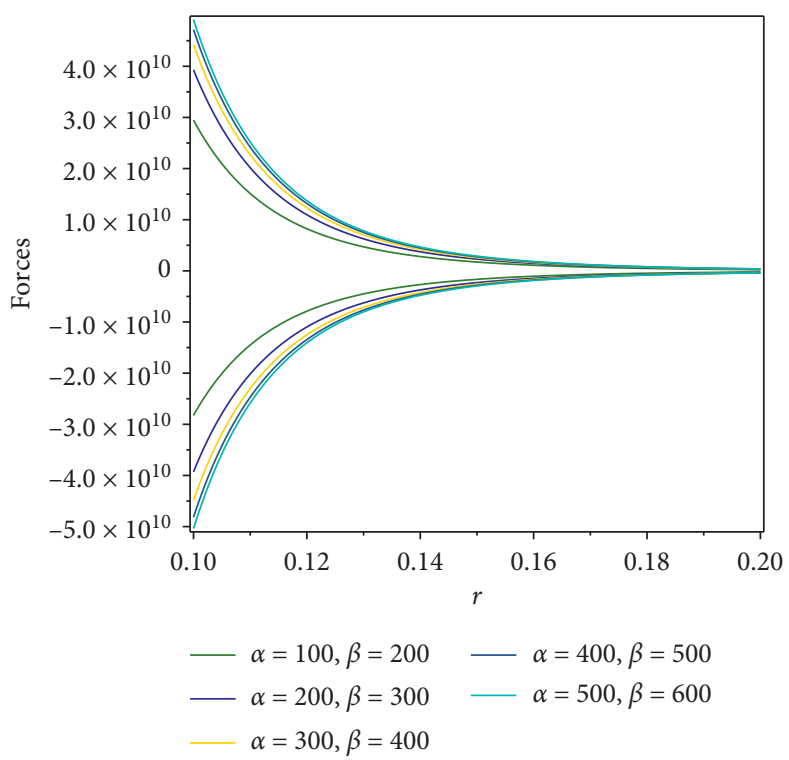

FIGURE 8: Behavior of hydrostatic and gravitational forces $\left(\mathscr{F}_{\mathscr{F}}, \mathscr{F}_{g}\right)$.

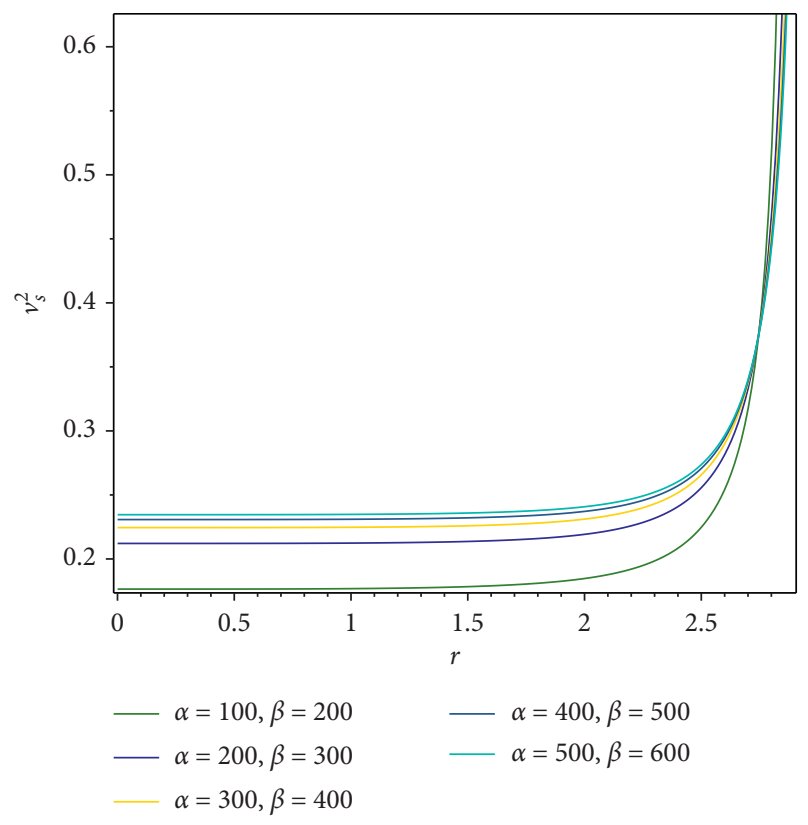

FIgURE 9: Variation of speed of sound.

everywhere and also have maximum value at the core of the star. Figure 5 indicates that all required energy bounds fulfill at every point inside the stellar structure and hence, it confirms the viability as well as consistency of our chosen EMSG model.

5.3. Compactness and Surface Redshift. The ratio between mass and radius of a stellar object is known as compactness factor. It can be seen clearly from the profile of the mass function given in Figure 6 that the mass of the star is directly proportional to the radius, and $M(r) \longrightarrow 0$ as $r \longrightarrow 0$, which shows that the mass function is regular at the center of the star. The compactness factor $u$ is defined as follows:

$$
u=\frac{M(r)}{r} \text {. }
$$

The gravitational redshift $\left(Z_{s}\right)$ acts as a crucial parameter to interpret the smooth relationship between particles in the celestial object. In the framework of compactness parameter, the gravitational redshift is expressed in the following form:

$$
Z_{s}=\frac{1}{\sqrt{1-2 u}}-1 \text {. }
$$

The graphical evolution of the compactness factor and surface redshift is given in Figure 7. These plots manifest that the behavior of $u(r)$ and $\left(Z_{s}\right)$ is increasing as required.

5.4. The Modified TOV Equation. The conservation equation is determined as follows:

$$
\nabla^{\mu} T_{\mu \nu}^{\mathrm{eff}}=0 .
$$

We investigate the equilibrium state of the compact stars through the modified TOV equation with dust matter configuration as follows:

$$
\frac{d p^{\mathrm{eff}}}{\mathrm{d} r}+\frac{\lambda^{\prime}}{2}\left(\rho^{\mathrm{eff}}+p^{\mathrm{eff}}\right)=0
$$

This equation determines the combination of two forces, i.e., hydrostatic force $\mathscr{F}_{\mathscr{F}}$ and gravitational force $\mathscr{F}_{q}$ that define the equilibrium state of the stellar structure. In the light of equation (41), these forces can be divided as $\mathscr{F}_{q}=$ $\left(\lambda^{\prime} / 2\right)\left(\rho^{\text {eff }}+p^{\text {eff }}\right)$ and $\mathscr{F}_{\mathscr{F}}=\left(d p^{\text {eff }} / \mathrm{d} r\right)$. The null impact of these forces $\left(\mathscr{F}_{\mathscr{F}}+\mathscr{F}_{q}=0\right)$ ensures the presence of physically realistic geometry of compact objects $[57,58]$. The graphical interpretation of $\mathscr{F}_{\mathscr{F}}$ and $\mathscr{F}_{g}$ for distinct values of $\alpha$ and $\beta$ is given in Figure 8, which shows that these forces 
counter-balance each other's effect and confirm the equilibrium state of our stellar system.

5.5. Stability Analysis. The stability of compact objects has great importance for a physically viable and consistent model. In order to check the stability of our considered model, we take into account Herrera's cracking method [59]. According to this technique, the square of sound speed $\left(v_{s}^{2}\right)$ must satisfy the condition $\left(0 \leq v_{s}^{2} \leq 1\right)$. The sound speed is determined as follows:

$$
v_{s}^{2}=\frac{d p^{\mathrm{eff}}}{\mathrm{d} \rho^{\mathrm{eff}}} .
$$

Figure 9 shows the graphical behavior of sound speed indicating that $v_{s}^{2}$ satisfies the required condition. This indicates the stability of our solution in this background.

\section{Concluding Remarks}

Noether symmetries are much helpful to find solutions of the dynamical system. These can also provide some viable conditions so that cosmological models can be selected according to current observations [60]. The Lagrange multipliers are used to minimize the dynamical system that ultimately helps to evaluate analytical solutions. In this paper, we have investigated the physical attributes of compact objects via Noether symmetry technique. For this purpose, we have taken static spherical spacetime with perfect fluid configuration in the context of EMSG. We have formulated the Lagrangian of this gravity and evaluated the symmetry generators with corresponding conserved quantities to analyze the solutions of modified equations of motion. The analytic solutions of Noether equations have been studied for the minimal coupling model of this theory by assuming dust fluid just for the sake of simplicity. The presence of conserved quantities is the key aspect in discussing the geometry of compact objects. The main findings of this analysis can be summarized as follows:

(i) For a physically realistic and stable model, $e^{\lambda}$ and $e^{\vartheta}$ must be positive, finite, and nonsingular everywhere inside the stellar structure. The graphical representation (Figures 1-3) of both the metric potentials shows the viability and stability of these quantities.

(ii) The effective matter variables must be maximum at the center of compact objects. We are unable to achieve the clear graphs for the complete range $(0 \leq r \leq 10)$ of radii. Therefore, we have plotted the graphs for the small radius to present the smooth behavior of compact objects. Figure 4 indicates that the effective matter variables have maximum value at the core of compact object and then decreases towards the surface boundary which shows physically viable behavior.

(iii) We have shown (Figure 5) that all energy conditions are well satisfied for our considered model exhibiting the physically viable matter. (iv) We have found (Figure 6) the direct proportionality of the mass function to the radius, $M(r) \longrightarrow 0$ as $r \longrightarrow 0$, suggesting that the mass function is regular at the center of the star.

(v) The graphical analysis of the compactness parameter and gravitational redshift function is found to be increasing as required (Figure 7).

(vi) It is found through TOV equation that gravitational $\mathscr{F}_{\mathscr{H}}$ and hydrostatic forces $\mathscr{F}_{\mathscr{G}}$ are in equilibrium for our proposed model (Figure 8) This ensures the stability of our system.

(vii) Finally, we have examined the causality condition through the speed of sound for the compact star. We have obtained (Figure 9) that our gravity model is consistent with this condition.

We have found that compact stars in this modified gravity via Noether symmetry technique depend on the first integrals of motion as well as model parameters $\alpha$ and $\beta$. We have shown that all physical characteristics of compact objects obey the physically viable pattern with small range of radii. We can conclude that Noether symmetry technique in the framework of energy-momentum squared gravity provides a physically realistic and stable model. It is worthwhile to mention here that this is the first investigation of compact objects through Noether symmetry technique in the energymomentum squared gravity [61].

\section{Data Availability}

Data sharing is not applicable to this article as no datasets were generated or analysed during the current study.

\section{Conflicts of Interest}

The authors declare that they have no conflicts of interest.

\section{References}

[1] J. Hanc, S. Tuleja, and M. Hancova, "Symmetries and conservation laws: consequences of Noether's theorem," American Journal of Physics, vol. 72, no. 4, p. 428, 2004.

[2] S. Capozziello, R. D. Ritis, and A. A. Marino, "Conformal equivalence and Noether symmetries in cosmology," Classical and Quantum Gravity, vol. 14, no. 12, p. 3259, 1997.

[3] S. Capozziello, G. Marmo, and C. P. Rubano, "Nöther symmetries in bianchi universes," IntInternational Journal of Modern Physics D, vol. 6, no. 4, p. 491, 1997a.

[4] U. Camci, "Approximate Noether gauge symmetries of the Bardeen model," European Physical Journal C, vol. 74, p. 3201, 2014.

[5] U. Camci and J. Cosmol, "Symmetries of geodesic motion in Gödel-type spacetimes," Journal of Cosmology and Astroparticle Physics, vol. 2014, no. 7, p. 2, 2014.

[6] A. D. Felice and S. R. Tsujikawa, " $f(R)$ theories," Living Reviews in Relativity, vol. 13, p. 3, 2010.

[7] S. i. Nojiri and S. D. Odintsov, "Unified cosmic history in modified gravity: from theory to Lorentz non-invariant models," Physics Reports, vol. 505, no. 2-4, p. 59, 2011. 
[8] K. S. Bamba, S. I. Nojiri, and S. D. Odintsov, "Dark energy cosmology: the equivalent description via different theoretical models and cosmography tests," Astrophysics and Space Science, vol. 342, no. 1, p. 155, 2012.

[9] T. Capozziello and F. Lobo, "Generalized curvature-matter couplings in modified gravity," Galaxies, vol. 2, no. 3, p. 410, 2014.

[10] T. Harko, T. S. Koivisto, and F. S. N. Lobo, "Palatini formulation of modified gravity with a non-minimal curvaturematter coupling," Modern Physics Letters A, vol. 26, no. 20, p. 1467, 2011.

[11] T. Harko, "Accelerating anisotropic cosmological model in $f(R, T)$ theory of gravity," Physical Review D, vol. 84, Article ID 024020, 2011.

[12] Z. Haghani, "Isotropic stars in higher-order torsion scalar theories," Physical Review D, vol. 88, Article ID 044023, 2013.

[13] P. H. R. S. Moraes and J. R. L. Santos, "A complete cosmological scenario from $f(R, T \varphi)$ gravity theory," European Physical Journal C, vol. 76, p. 60, 2016.

[14] N. Katirci and M. Kavuk, "Constraint on energy-momentum squared gravity from neutron stars," European Physical Journal-Plus, vol. 129, p. 163, 2014.

[15] C. V. R. Board and J. D. Barrow, "Low redshift constraints on energy-momentum-powered," Physical Review D, vol. 96, Article ID 123517, 2017.

[16] N. Nari and M. Roshan, "Compact stars in energy-momentum squared gravity," Physical Review D, vol. 98, Article ID 024031, 2018.

[17] P. H. R. S. Moraes and P. K. Sahoo, "Non-exotic matter wormholes in a trace of the energy-momentum tensor squared gravity," Physical Review D, vol. 97, Article ID 024007, 2018.

[18] O. Akarsu, "Constraint on energy-momentum squared gravity from neutron stars and its cosmological implications," Physical Review D, vol. 97, Article ID 124017, 2018.

[19] S. Bahamonde, M. Marciu, and P. Rudra, "Dynamical system analysis of generalized energy-momentum-squared gravity," Physical Review D, vol. 100, Article ID 083511, 2019.

[20] C. Y. Chen and P. Chen, "Eikonal black hole ringings in generalized energy-momentum squared gravity," Physical Review D, vol. 101, Article ID 064021, 2020.

[21] S. Bhattacharjee and P. K. Sahoo, "Big bang nucleosynthesis and entropy evolution in $f(R, T)$ gravity," The European Physical Journal Plus, vol. 135, p. 86, 2020.

[22] A. H. Barbar, A. M. Awad, and M. T. AlFiky, "Viability of bouncing cosmology in energy-momentum-squared gravity," Physical Review D, vol. 101, Article ID 044058, 2020.

[23] S. Capozziello, A. Stabile, and A. Troisi, "Spherical symmetry in $f(R)$-gravity," Classical and Quantum Gravity, vol. 25, Article ID 085004, 2008.

[24] M. Roshan and F. Shojai, "Palatini $f(R)$ cosmology and Noether symmetry," Physics Letters B, vol. 668, pp. 238-240, 2008.

[25] I. Hussain, M. Jamil, and F. M. Mahomed, "Noether gauge symmetry approach in $f(R)$ gravity," Astrophysics and Space Science, vol. 337, no. 1, p. 373, 2012.

[26] Y. Kucukakca, "Scalar tensor teleparallel dark gravity via Noether symmetry," European Physical Journal C, vol. 73, p. $2327,2013$.

[27] S. Bahamonde, "Cambridge Repository," Physical Review D, vol. 94, Article ID 084042, 2016.

[28] M. Sharif and H. Ismat Fatima, "Noether symmetries in $f(G)$ gravity," Journal of Experimental and Theoretical Physics, vol. 122, no. 1, p. 104, 2016.
[29] M. F. Shamir and M. Ahmad, "Emerging anisotropic compact stars in $f(G, T)$ gravity," European Physical Journal C, vol. 77, p. 55, 2017.

[30] M. F. Shamir and M. Ahmad, "Some exact solutions $f(G, T)$ in gravity via Noether symmetries," Modern Physics Letters A, vol. 32, no. 16, p. 1750086, 2017a.

[31] M. Sharif and S. Waheed, "Re-scaling of energy in stringy charged black hole solutions using approximate symmetries," Canadian Journal of Physics, vol. 88, no. 11, p. 833, 2010.

[32] M. Sharif and S. Waheed, "Energy of the bardeen model using an approximate symmetry method," Physica Scripta, vol. 83, Article ID 015014, 2011.

[33] M. Sharif and I. Nawazish, "Noether symmetries in $f(G)$ gravity," Journal of Experimental and Theoretical Physics, vol. 120, p. 49, 2014.

[34] M. Sharif and I. Shafique, "Noether symmetries in a modified scalar-tensor gravity," Physical Review D, vol. 90, Article ID 084033, 2014.

[35] M. Sharif and M. Z. Gul, "Study of charged spherical collapse in $f(G, T)$ gravity," European Physical Journal - Plus, vol. 133, p. $345,2018$.

[36] M. Sharif and M. Z. Gul, "Dynamics of perfect fluid collapse in $f(G, T)$ gravity," International Journal of Modern Physics D, vol. 28, no. 3, p. 1950054, 2019.

[37] M. Sharif and M. Zeeshan Gul, "Dynamics of cylindrical collapse in $f(G, T)$ gravity," Chinese Journal of Physics, vol. 57, p. 329, 2019a.

[38] Y. Kucukakca and A. R. Akbarieh, "Noether symmetries of Einstein-aether scalar field cosmology," European Physical Journal C, vol. 80, p. 1019, 2020.

[39] M. F. Shamir and T. Naz, "Stellar structures in $f(G)$ gravity admitting Noether symmetries," Physics Letters B, vol. 806, p. 135519, 2020.

[40] A. Malik, M. F. Shamir, and I. Hussain, "Noether symmetries of LRS Bianchi type-I spacetime in $f(R, \varphi, \chi)$ gravity," International Journal of Geometric Methods in Modern Physics, vol. 17, no. 11, p. 2050163, 2020.

[41] G. Mustafa, X. Tie-Cheng, and M. F. Shamir, "Realistic solutions of fluid spheres in $f(G, T)$ Gravity under Karmarkar condition," Annals of Physics, vol. 413, p. 168059, 2020.

[42] G. Mustafa, M. F. Shamir, and X. Tie-Cheng, "Physically viable solutions of anisotropic spheres in $f(R, G)$ gravity satisfying the Karmarkar condition," Physical Review D, vol. 101, p. 104013, 2020a.

[43] M. Camenzind, Compact Objects in Astrophysics, Springer, Berlin, Germany, 2007.

[44] G. Abbas, A. Kanwal, and M. Zubair, "Anisotropic strange quintessence stars in $f(T)$ gravity," Astrophysics and Space Science, vol. 357, p. 109, 2015.

[45] M. Sharif and F. Javed, "On the stability of bardeen thin-shell wormholes," General Relativity and Gravitation, vol. 48, p. 158, 2016.

[46] G. Abbas, "Anisotropic compact stars in $f(G)$ gravity," Astrophysics and Space Science, vol. 357, p. 158, 2015a.

[47] M. Zubair and G. Abbas, "Some interior models of compact stars in $f(R)$ gravity," Astrophysics and Space Science, vol. 361, p. 342, 2016.

[48] A. V. Astashenok, S. Capozziello, and S. D. Odintsov, "Dynamical analysis of self-gravitating stars in $f(R, T)$ gravity," Physical Review D, vol. 89, p. 103509, 2014.

[49] A. V. Astashenok, S. Capozziello, and S. D. Odintsov, "Extreme neutron stars from extended theories of gravity," Journal of Cosmology and Astroparticle Physics, vol. 2015, no. 1, 2015. 
[50] A. V. Capozziello, S. Capozziello, and S. D. Odintsov, "Nonperturbative models of quark stars in $\mathrm{f}(\mathrm{R})$ gravity," Physics Letters B, vol. 742, p. 160, 2015 a.

[51] S Capozziello, "Mass-radius relation for neutron stars in $f(R)$ gravity," Physical Review D, vol. 93, Article ID 023501, 2016.

[52] D. Momeni, H. Gholizade, and R. Myrzakulov, "Mimetic compact stars," International Journal of Geometric Methods in Modern Physics, vol. 15, no. 6, p. 1850091, 2018.

[53] K. D. Moraes and J. Barua, "A singularity-free solution for a charged fluid sphere in general relativity," Journal of Physics A: Mathematical and General, vol. 8, no. 4, p. 508, 1975.

[54] S. Capozziello, A. Stabile, and A. Troisi, "Spherically symmetric solutions in $f(R)$ gravity via the Noether symmetry approach," Classical and Quantum Gravity, vol. 24, no. 8, p. 2153, 2007.

[55] S. Capozziello, M. De Laurentis, and A. Stabile, "Axially symmetric solutions in $f(R)$-gravity," Classical and Quantum Gravity, vol. 27, no. 16, p. 165008, 2010.

[56] J. Santos, "Energy conditions in $f(R)$ gravity," Physical Review $D$, vol. 76, Article ID 083513, 2007.

[57] J. R. Oppenheimer and G. M. Volkoff, "On massive neutron cores," Physical Review, vol. 55, no. 4, p. 374, 1939.

[58] R. C. Tolman, "Static solutions of einstein's field equations for spheres of fluid," Physical Review, vol. 55, no. 4, p. 364, 1939.

[59] L. Herrera, "Cracking of self-gravitating compact objects," Physics Letters A, vol. 165, no. 3, p. 206, 1992.

[60] S. Capozziello, M. Laurentis, and S. D. Odintsov, "Noether symmetries in Gauss-Bonnet-teleparallel cosmology," European Physical Journal C, vol. 72, p. 1434, 2012.

[61] D. Momeni, H. Gholizade, M. Raza, and R. Myrzakulov, "Tolman-Oppenheimer-Volkoff equations in nonlocal $f(R)$ gravity," International Journal of Modern Physics A, vol. 30, no. 16, Article ID 1550093, 2015. 